\title{
Controversias sobre el tamizaje de cáncer mamario con mamografía
}

\author{
Controversies about breast cancer screening with mammography
}

María Belén Doval $^{a}$, Camila Volij ${ }^{a}$, Mariela Weisbrot ${ }^{a, b, c}$, Sergio Terrasa $a^{a, d, b}$

\begin{abstract}
Resumen
Al igual en otras partes del mundo, en Argentina la mortalidad específica por cáncer mamario disminuyó de forma sostenida entre 1996 y 2017. Es probable que en la actualidad la mamografía sea la herramienta más importante para detectar precozmente, evaluar y llevar un seguimiento de las personas con esta patología. Sin embargo existe mucha controversia acerca de los beneficios del tamizaje poblacional y de la realización de diagnósticos precoces de cáncer de mama. El propósito de esta revisión narrativa es brindar una mirada contraintuitiva, cuestionadora de los beneficios de esta práctica, ponderando también sus riesgos, escasamente visibilizados por las estrategias comunicacionales concordantes con el modelo de prevenir es mejor que curar y la lucha contra la historia natural del cáncer.

Abstract

As in other parts of the world, in Argentina, breast cancer-specific mortality declined steadily between 1996 and 2017. Mammography is currently the most important tool for early detection, evaluation and follow-up of people suffering from this disease. However, there is a controversy about the benefits of population screening and early diagnosis of breast cancer. The aim of this narrative review is to provide a counterintuitive, questioning view of the benefits of this practice, also weighing its risks, poorly visible through communication strategies consistent with the model of prevention is better than cure and the fight against the natural history of cancer.
\end{abstract}

Palabras clave: Tamizaje Masivo, Neoplasias de la Mama, Servicios Preventivos de Salud, Toma de Decisiones. Keywords: Mass Screening, Breast Neoplasms, Preventive Health Services, Decision Making.

Doval MB, Volij C, Weisbrot M, Terrasa S.. Controversias sobre el tamizaje de cáncer mamario con mamografía. Evid Actual Pract Ambul. 2019;22(3):e002023.

\section{Introducción}

Al igual que lo que sucede en otras partes del mundo, el Ministerio de Salud de la República Argentina comunicó que la mortalidad específica por cáncer mamario continúa disminuyendo de forma sostenida entre 1996 y 2017. En sintonía con lo que sostienen otros expertos ${ }^{1-3}$, los autores de este artículo consideramos que una buena fracción de este descenso estaría vinculado, principalmente, con la mejora de la terapéutica para esta enfermedad y con la especialización de los centros de tratamiento de cáncer de mama, existiendo un gran debate respecto de la influencia de los programas masivos de rastreo.

Es probable que la mamografía sea la herramienta más importante que tienen los profesionales de la salud para detectar precozmente el CM y también para diagnosticar, evaluar y realizar un seguimiento de las personas con esta patología. Sin embargo, en la actualidad existe una gran controversia respecto de los beneficios y los riesgos del tamizaje del $\mathrm{CM}$, de la mejor edad para iniciarlo, del intervalo óptimo entre los estudios y del número necesario de mujeres para prevenir un episodio de CM.

\section{El debate sobre el impacto sanitario de los programas de rastreo de cáncer mamario}

Posturas que destacan el impacto sanitario de los programas de rastreo de cáncer mamario

Algunos autores sostienen que las estrategias de rastreo con mamografía previenen una muerte por cáncer de mama por cada 235 personas tamizadas ${ }^{4}$ y confieren una reducción de la mortalidad del $40 \%{ }^{5}$.
Por ejemplo, la Agencia Internacional para la Investigación del Cáncer ${ }^{6}$ sostiene que hace 35 años existe evidencia proveniente de ensayos clínicos aleatorizados de que las estrategias de rastreo de CM a través de mamografía reducen $23 \%$ la mortalidad por esta enfermedad ${ }^{7}$

Mardsen et $\mathrm{al}^{8}$ documentaron en Europa un descenso de la mortalidad por CM cercano a un $35 \%$ durante las últimas tres décadas, adjudicando este logro a las mejoras en la implementación del rastreo de esta condición, al mejor manejo del CM y al menor uso de terapia de reemplazo hormonal.

La postura del Instituto Nacional del Cáncer (INC) de Argentina ${ }^{9}$ es concordante con la de la Fuerza de Tareas de Servicios Preventivos de EE.UU. (en inglés, USPSTF), que destaca que los beneficios de la reducción de la mortalidad por CM atribuibles a los programas de rastreo varían en función del rango etario de las destinatarias. Se prevendrían 2,9 (Intervalo de confianza [IC] del $95 \%$ : - 0,6 a 9,9) muertes por cada 10.000 mujeres entre los 39 y los 49 años de edad sometidas a tamizaje mamográfico bianual durante diez años; 7,7 (IC 95\%:1,6 a 17,2) muertes cuando el cribado es realizado entre los 50 y los 59 años; 12,5 (IC 95\%: 2 a 32,1) entre los 60 y 69 años; y 21,3 (IC 95\%: 10,7 a 31,7) entre los 70 y los 74 años ${ }^{10}$.

Posturas que cuestionan el impacto sanitario de los programas de rastreo de cáncer mamario

En 2000, el Grupo Nórdico de la Colaboración Cochrane publicó un artículo ${ }^{11}$ que resaltaba la gran incertidumbre respecto de la magnitud del efecto de la mamografía en la reducción de la mortalidad por CM, dado que consideraba metodológicamente

\footnotetext{
a Servicio de Medicina Familiar y Comunitaria, Hospital Italiano de Buenos Aires. maria.doval@hospitalitaliano.org.ar

b Departamento de Salud Pública, Instituto Universitario Hospital Italiano.

c Departamento de Investigación, Instituto Universitario Hospital Italiano.

d Departamento de Investigación, Hospital Italiano de Buenos Aires.
} 
inaceptables los metanálisis que apoyaban dicha postura. Esto tuvo una enorme resonancia mundial y otras voces se hicieron eco de esta polémica.

En 2008 Becker et al $^{12}$ calcularon que sería necesario que entre 10.000 y 20.000 mujeres realicen una mamografía para evitar una muerte por $\mathrm{CM}$; por otra parte, Park et $\mathrm{al}^{13}$ sostienen que el $80 \%$ de la reducción en mortalidad observada entre 1973 y 2010 en EE.UU se debería al mejor manejo de las pacientes y no al efecto del tamizaje masivo.

En consonancia, otros expertos ${ }^{14-16}$ concluyeron que los principales factores para la reducción de la mortalidad de $\mathrm{CM}$ en Europa, Australia y EE.UU. durante los últimos 25 años habrían sido los tratamientos adyuvantes y la quimioterapia tras la cirugía inicial.

El impacto de la reducción de la mortalidad de programas de rastreo se mide a través de la disminución de la incidencia del número de cánceres avanzados. Por lo tanto, en lugares donde estos programas tienen una importante cobertura, debería haber habido menor número de cánceres avanzados. No obstante, en contra de lo que habría de esperarse, en las regiones donde la mamografía se realiza desde hace muchos años con gran penetrancia, se observó una escasa o nula disminución de la incidencia de cánceres avanzados, incluyendo los metastásicos de novo (estadio IV) ${ }^{17}$.

Los programas de rastreo masivo fueron implementados en distintos momentos históricos en diferentes países y regiones. Basándonos en la argumentación de Autier et al ${ }^{17}$, tomaremos como ejemplo EE.UU., Noruega y Suiza, que son los tres países de la Organización para la Cooperación y el Desarrollo Económico (OCDE) con mayor gasto en salud per cápita y mejor difusión de las nuevas terapias médicas. En EE.UU. los programas de rastreo masivo fueron introducidos en 1980, mientras que en Noruega comenzaron en 1995 y en los cantones de Suiza, continúa existiendo una penetrancia baja de tamizaje de CM $35 \%$ en 2012). En aquellas regiones con implementación masiva de dichos programas de rastreo, la reducción de la mortalidad por CM fue similar a la de los lugares de baja penetrancia y tardía introducción del rastreo.

Por otro lado, en general no se contempla el daño causado por las mamografías realizadas en el contexto de programas masivos de rastreo ${ }^{18} \mathrm{y}$, asimismo, la potencial reducción en las tasas de mortalidad por CM atribuidas al rastreo continúan siendo cuestionadas -y hasta podrían ser anuladas- por las consecuencias del sobrediagnóstico, la mortalidad relacionada a cirugías y tratamientos de quimio y radioterapia ${ }^{19,20}$, la terapia anti-estrogénica y el tratamiento neoadyuvante ${ }^{21}$ potencialmente innecesario ${ }^{19}$.

Existe una gran variación de la estimación de la proporción de los diagnósticos de $\mathrm{CM}$ que podrían corresponder a sobrediagnósticos. Mientras que el Panel Independiente sobre Cáncer de Mama del Reino Unido ${ }^{22}$ estima que $19 \%$ de los cánceres diagnosticados en mujeres invitadas a realizar tamizaje pueden ser interpretados como eventos de sobre-detección, el metaanálisis publicado por Jacklyn et al ${ }^{23}$ comunicó que la proporción de sobrediagnóstico podría llegar a $30 \%$ en el contexto de programas masivos de rastreo. Vale destacar que Loberg et al ${ }^{19}$ sostienen que las discordancias en estas estimaciones podrían ser consecutivas a los diferentes denominadores empleados en la medición.

\section{¿Prevenir es mejor que curar?}

La idea de que prevenir es mejor que curar ha dominado la medicina durante el último medio siglo, y la frase suele utilizarse para avalar muchas intervenciones en pos de la detección pre$\mathrm{coz}$ de diversas condiciones clínicas. Sin embargo, no siempre la detección temprana redunda en una verdadera mejora de la calidad de vida de la persona. Por ejemplo, a pesar de que más de $70 \%{ }^{24}$ de las mujeres de EE.UU. realiza cribado mamográfico, continuaron aumentando las mastectomías radicales sin mayor descenso en la mortalidad por $\mathrm{CM}^{25}$.

Asimismo, la detección precoz de carcinomas ductales in situ (CDIS) (cerca de $20 \%$ del total de los CM diagnosticados) y su tratamiento precoz (radioterapia o mastectomía), no mostraron reducir la mortalidad específica por $\mathrm{CM}$ ni la recurrencia de tumor invasivo ${ }^{26}$. Por otro lado, antes de la era del rastreo de CM, los CDIS representaban menos de $5 \%$ de todos los CM, mientras que tras de la difusión de estos programas, hoy representan entre 15 y $20 \%{ }^{27}$. Este dramático incremento, asociado a la remoción quirúrgica y a la radioterapia para prevenir la recurrencia, no se acompañó de un descenso de la incidencia de cánceres invasivos o de disminución en la mortalidad por $\mathrm{CM}^{28}$

Es por eso que consideramos que los posibles beneficios del cribado deben ser contrapuestos con los posibles daños vinculados al sobrediagnóstico, que según Morris, podrían llegar incluso hasta $50 \%$ de los casos detectados, y derivar en la necesidad de mayor número de tratamientos ${ }^{29}$.

Como acabamos de exponer, y como sostienen Spina et $\mathrm{al}^{30}$, las estrategias de prevención secundaria del cáncer no siempre son inocuas. Sus potenciales daños incluyen: a) resultados falsamente positivos; b) efectos adversos de los exámenes complementarios (p. ej. infección del sitio de una punción mamaria); c) sobrediagnóstico, entendido como el correcto diagnóstico de una condición clínica (en este caso, un CM) en el marco de un cuadro que no habría causado daño alguno a la paciente, quien tras el diagnóstico queda etiquetada como una persona que sufre de cáncer, con la preocupación consiguiente; y d) sobretratamiento, en caso de ocurrir un sobrediagnóstico de cáncer de mama, con los riesgos que eso conlleva.

\section{Las dificultades para desimplementar estas prácticas}

Existe preocupación en la comunidad científica y en quienes tienen posiciones gubernamentales en torno al grave problema que representan los estudios y procedimientos innecesarios ${ }^{31}$. En relación a las estrategias de rastreo masivo de $\mathrm{CM}$, se reconoce al sobrediagnóstico como el mayor riesgo ${ }^{22}$, vinculado con la edad de inicio del estudio y al intervalo en el que se realiza.

A partir del conocimiento sobre los riesgos que conllevan las mamografías, sus resultados falsos positivos y los sobrediagnósticos a los que pueden conducir (en especial, cuando son realizadas a edades tempranas), en 2009, la USPSTF y la Fuerza de Tareas Preventivas de Canadá (CTFPH) comenzaron a recomendar retrasar el inicio del rastreo y aumentar su intervalo a dos años (desde iniciar la mamografía a los 40 años y realizarla anualmente, a comenzar a los 50 años con una frecuencia bienal, terminándose a los 70 años) ${ }^{10}$. Probablemente por una mala estrategia comunicacional, Allen et al ${ }^{32}$ documentaron en EE.UU. que la respuesta de las mujeres a esta nueva recomendación fue de desconcierto y desconfianza, debido a la sospecha de que este consejo tuviera como única finalidad reducir los costos de los programas de rastreo.

Por otro lado, Buck et al ${ }^{33}$ documentaron en Canadá que $22 \%$ de las mujeres de 40 a 49 realizaron mamografías de tamizaje; mientras que un estudio en Argentina realizado por nuestro equipo de trabajo reportó que 52,8\% (IC 95\%: 45,6 a 59,9) de las mamografías realizadas antes de los 40 años habían sido solicitadas por este motivo ${ }^{34}$

La dificultad para adaptarse a las nuevas recomendaciones sobre el rastreo del CM se refleja en las encuestas realizadas a distintos profesionales de la salud: médicos de familia, clínicos y ginecólogos manifestaron gran resistencia al cambio ${ }^{35}$. El $80 \%$ 
continúa realizando cribado mamográfico entre los 40 y los 44 años, y $88 \%$, continúa recomendando esta práctica en mujeres de 45 a 49 años, aún conociendo que existe evidencia en contra de realizarla y estando al tanto de las nuevas recomendaciones de la USPSTF.

\section{La influencia de las estrategias comunicacionales para construir posturas a favor del rastreo}

Gummersbach et $\mathrm{al}^{3}$ revisaron la información provista en los folletos de promoción del tamizaje de cáncer de mama difundidos en Alemania, España, Italia y Francia, concluyendo que carecían de información esencial para que sus destinatarias pudieran realizar una decisión informada.

En Argentina, un estudio realizado por nuestro grupo en 2015 documentó que $95 \%$ de la información difundida por los cinco sitios web de los diarios de mayor tráfico no era concordante con las recomendaciones del INC, ya que promovía la realización de mamografía desde los 40 años (cuando este organismo la recomienda a partir de los 50 años) y el autoexamen mamario. Además, los principales avales citados no estaban basados en la evidencia científica sino en las posturas de organizaciones civiles, empresas y fundaciones que realizan campañas contra el $\mathrm{CM}^{30}$.

\section{Percepciones, actitudes y entendimiento de las usua- rias respecto del tamizaje de cáncer mamario}

En Argentina, una investigación realizada por nuestro equipo, que indagó los factores que motivan a las mujeres a realizar mamografías de tamizaje en el Hospital Italiano de Buenos Aires permitió comprender que la motivación se construye sobre tres grandes pilares: a) el dogma de que prevenir es mejor que curar, b) la asimetría en la relación Médico-Paciente en el contexto de un modelo médico hegemónico, y c) una fuerte impronta de los medios de comunicación, que remarcan los beneficios de la técnica sin difundir sus riesgos ${ }^{36}$. Los mensajes de los líderes de opinión pública transmiten la importancia de "cuidarse", "protegerse" y "tomar conciencia", lo que convierte a la realización de la mamografía en una obligación cuasi moral ${ }^{36}$.

Por otro lado, un trabajo realizado en Australia documentó que las mujeres pueden modificar su decisión de realizarse mamografías de rastreo de acuerdo con la tasa de sobrediagnóstico que se les informe ${ }^{37}$.

Una revisión sistemática ${ }^{38}$ evaluó las perspectivas y el conocimiento acerca de los potenciales efectos negativos de la mamografía, concluyendo que existe un limitado entendimiento por parte de las usuarias del sistema de salud en torno al concepto de sobrediagnóstico. Más aún, luego de que se les explicara este concepto, sólo $10 \%$ de las participantes logró comprenderlo adecuadamente y la mayoría de las mujeres manifestó que estaría agradecida de enterarse de un resultado de rastreo mamográfico positivo, independientemente de que éste fuera verdadero o falso. Además, luego de recibir la intervención informativa sobre el significado de los resultados falsos positivos, $97 \%$ de las participantes piensa que continuaría realizando la práctica preventiva.

\section{Los procesos de toma de decisiones compartidas}

Cotidianamente nos enfrentamos con la necesidad de definir conductas preventivas o terapéuticas en el marco de nuestra práctica clínica. Por lo general, existe más de una opción posible, y cada escenario implica ventajas y desventajas.

Como resultado de las diferentes interpretaciones de la evidencia en torno a los potenciales beneficios y daños del tamizaje de
CM y las discrepancias en las recomendaciones en los distintos países, surge la necesidad de implementar procesos de toma de decisiones compartidas (TDC) entre las usuarias del sistema de salud y los profesionales que las asisten.

Así como las personas toman las decisiones importantes de su vida teniendo conocimiento de los pros y contras de cada opción y poniendo en juego sus valores y preferencias, sería deseable que tuviesen en cuenta los mismos recaudos en los cuidados de su salud ${ }^{39}$. En esta línea de pensamiento, las decisiones más eficaces, tanto para los usuarios como para los sistemas de salud, son aquellas que se toman teniendo en cuenta los valores y preferencias de las pacientes, la evidencia científica y el contexto.

Una revisión sistemática ${ }^{40}$ que había incluido 105 estudios, con un total de 31.043 participantes, y evaluado 50 tipos de decisiones diferentes como cirugías y tamizajes de diferentes neoplasias (cáncer de mama, próstata y colon) documentó que las herramientas estructuradas para la ayuda en la toma de decisiones compartidas optimizan estos procesos en términos de reducir significativamente el número de participantes indecisos. Asimismo, luego de esta interacción aumenta la comunicación positiva entre pacientes y profesionales de la salud, promoviendo la satisfacción en torno a las decisiones tomadas y facilitando el rol activo de las personas.

Ivley et $\mathrm{al}^{41}$ evaluaron las intenciones de las mujeres de 38 a 50 años de discontinuar el tamizaje de $\mathrm{CM}$ o de no iniciarlo, tras haber sido expuestas a un proceso de TDC implementado a través de herramientas de ayuda (decision aids), y encontraron que $77 \%$ de las participantes decidiría no iniciar y/o continuar con el rastreo luego de esta intervención.

En otra investigación, Hersch et $\mathrm{al}^{42}$ compararon dos herramientas de ayuda en la TDC en rstreo de CM que difieren únicamente en si le brindan o no a la usuaria información acerca de sobrediagnóstico. Concluyeron que aquellas que estuvieron expuestas a esa información adicional tuvieron una menor actitud positiva respecto del realizar mamografía respecto a quienes no la recibieron.

\section{A modo de conclusión}

Por todo lo expresado, podemos concluir que las usuarias de los sistemas de salud habitualmente desconocen: 1) que la reducción de la mortalidad por $\mathrm{CM}$ atribuible a los programas de rastreo estaría sobreestimada; 2) las implicancias de los resultados falsos positivos y/o del sobrediagnóstico; 3) que las recomendaciones de tamizaje de CM se han vuelto más conservadoras (inicio más tardío y periodicidad más amplia); 4) los conflictos de intereses de algunos de los impulsores de las estrategias comunicacionales de prevención del CM, (p. ej. industria farmacéutica, fabricantes de implantes, centros de diagnóstico radiológico, etc).

Además, a los profesionales de la salud todavía nos resulta difícil comprender los conceptos vinculados al sobrediagnóstico y por lo tanto, más aún, explicarlos. Si a esto le sumamos nuestro miedo al litigio y que en nuestra sociedad existe una mayor comprensión de los riesgos de no realizar diagnósticos que los vinculados a sobrediagnosticar, la situación se complica aún más.

Los profesionales de la salud tenemos la responsabilidad de facilitar que las toma de decisiones vinculadas con la salud de nuestras pacientes sean compartidas. Sin embargo, esta tarea no resulta sencilla ya que, por un lado, estamos atravesados por la misma cultura que ellas, y por otro, porque el paternalismo médico sigue primando en nuestra sociedad.

\section{Referencias}

1. Nystrm L, Andersson I, Bjurstam N, Frisell J, Nordenskjöld B, Rutqvist LE. Long-term effects of mammography screening: updated overview of the Swedish randomised trials. The Lancet. 2002;359(9310):909-919. Available from: 10.1016/S0140-6736(02)08020-0. 
2. Barratt A, Howard K, Irwig L, Salkeld G, Houssami N. Model of outcomes of screening mammography: information to support informed choices. BMJ. 2005;330(7497):936-936. Available from: 10.1136/bmj.38398.469479.8F.

3. Gummersbach E, Piccoliori G, Zerbe CO, Altiner A, Othman C, Rose C, et al. Are women getting relevant information about mammography screening for an informed consent: a critical appraisal of information brochures used for screening invitation in Germany, Italy, Spain and France . Eur J Public Health. 2010;24(4):409-414. Available from: 10.1093/eurpub/ckp174.

4. Marmot MG, Altman DG, Cameron DA, Dewar JS, Thompson SG, Wilcox M. The benefits and harms of breast cancer screening: an independent review. Br J Cancer. 2013;108(11):2205-2240. Available from: 10.1038/bjc.2013.177.

5. Helvie MA, Chang JT, Hendrick RE, Banerjee M. Reduction in late-stage breast cancer incidence in the mammography era: Implications for overdiagnosis of invasive cancer. Cancer. 2014;120(17):2649-2656. Available from: 10.1002/cncr.28784.

6. Lauby-Secretan B, Loomis D, Straif K. Breast-Cancer Screening - Viewpoint of the IARC Working Group. New England Journal of Medicine. 2015;373(15):2353-2358. Available from: 10.1056/NEJMc1508733.

7. Nelson HD, Fu R, Cantor A, Pappas M, Daeges M, Humphrey L. Effectiveness of Breast Cancer Screening: Systematic Review and Meta-analysis to Update the Preventive Services Task Force Recommendation. Ann Intern Med. 2009;164(4):244-255. Available from: 10.7326/M15-0969.

8. Marsden J, Hamoda $\mathrm{H}$. European cancer mortality predictions for the year 2019 with focus on breast cancer [Epub ahead of print]. Ann Oncol. 2019;30. Available from: 10.1093/annonc/mdz051.

9. Instituto Nacional del Cáncer . Cáncer de mama; 2018. Available from: http://www.msal.gov.ar/inc/acerca-del-cancer/cancer-de-mama/ [Last access: 2019-12-11].

10. US Preventive Services Task Force. Screening for Breast Cancer: US Preventive Services Task Force Recommendation Statement. Ann Intern Med. 2009;151(10):716-726. Available from: 10.7326/0003-4819-151-10-200911170-00008.

11. Gøtzsche PC, Olsen O. Is screening for breast cancer with mammography justifiable? The Lancet. 2000;355(9198):129-134. Available from: 10.1016/S0140-6736(99)06065-1[IndexedforMEDLINE].

12. Becker N, Junkermann H. MEDIZIN-Ubersichtsarbeit-Nutzen und Risiko des Mammografie-Screenings-Betrachtungen aus epidemiologischer Sicht. Deutsches Arzteblatt-Arztliche Mitteilungen-Ausgabe. 2008;105(8):131-136. Available from: 10.3238/arztebl.2008.0131.

13. Park JH, Anderson WF, Gail MH. Improvements in US Breast Cancer Survival and Proportion Explained by Tumor Size and Estrogen-Receptor Status. Journal of Clinical Oncology. 2015;33(26):2870-2876. Available from: 10.1200/JCO.2014.59.9191.

14. Bleyer A, Welch HG. Effect of Three Decades of Screening Mammography on Breast-Cancer Incidence. New England Journal of Medicine. 1998;367(21):1998-2005. Available from: 10.1056/NEJMoa1206809.

15. Autier P, Boniol M, Gavin A, Vatten LJ. Breast cancer mortality in neighbouring European countries with different levels of screening but similar access to treatment: trend analysis of WHO mortality database. BMJ. 2011;343:d4411. Available from: 10.1136/bmj.d4411.

16. Beral V, Hermon C, Reeves G, Peto R. Sudden fall in breast cancer death rates in England and Wales. Lancet. 1995;345(8965):1642-1643. The LancetHYPERLINK. Available from: 10.1016/s0140-6736(95)90151-5.

17. Autier P, Boniol M. Mammography screening: A major issue in medicine. European Journal of Cancer. 2018;90:34-62. Available from: 10.1016/j. ejca.2017.11.002.

18. Miglioretti DL, Lange J, van den Broek J, Lee C, van Ravesteyn N, Ritley D, et al. Radiation-Induced Breast Cancer Incidence and Mortality From Digital Mammography Screening. Annals of Internal Medicine. 2016;164(4):205-205. Available from: 10.7326/M15-1241.

19. Løberg M, Lousdal ML, Bretthauer M, Kalager M. Benefits and harms of mammography screening. Breast Cancer Research. 2015;17(1):63. Available from: 10.1186/s13058-015-0525-Z.

20. Darby SC, Ewertz M, McGale P, Bennet AM, Blom-Goldman U, Brønnum D, et al. Risk of ischemic heart disease in women after radiotherapy for breast cancer. N Engl J Med. 2013;368(11):987-998. Available from: 10.1056/NEJMoa1209825.

21. Yeh ET, Bickford CL. Cardiovascular Complications of Cancer Therapy: incidence, pathogenesis, diagnosis, and management. Journal of the American College of Cardiology. 2009;53(24):2231-2247. Available from: 10.1016/j.jacc.2009.02.050.

22. Independent UK Panel on Breast Cancer Screening. The benefits and harms of breast cancer screening: an independent review. Lancet. 2012;380(9855):1778-1786. Available from: 10.1016/S0140-6736(12)61611-0.

23. Jacklyn G, Glasziou P, Macaskill P, Barratt A. Meta-analysis of breast cancer mortality benefit and overdiagnosis adjusted for adherence: improving information on the effects of attending screening mammography. Br J Cancer. 2016;114(11):1269-1276. Available from: 10.1038/bjc.2016.90.

24. Hall FM. Guidelines for Screening Mammography. J Am Coll Radiol. 2018;15(1):8-9. Available from: 10.1016/j.jacr.2017.09.033.

25. Gøtzsche PC, Jørgensen KJ. Screening for breast cancer with mammography. Cochrane Database of Systematic Reviews. 2013;(6). Available from: 10.1002/14651858.cd001877.pub5.

26. Barrio AV, Zee KJV. Controversies in the Treatment of Ductal Carcinoma in Situ. Annu Rev Med. 2017;68:197-211. Available from: 10.1146/annurevmed-050715-104920.

27. Punglia RS, Schnitt SJ, Weeks JC. Treatment of ductal carcinoma in situ after excision: would a prophylactic paradigm be more appropriate? J Natl Cancer Inst. 2013;105(20):1527-1533. Available from: 10.1093/jnci/djt256.

28. Narod SA, labal J, Giannakeas V, Sopik V, Sun P. Breast Cancer Mortality After a Diagnosis of Ductal Carcinoma In Situ. JAMA Oncol. 2015;1(7):888896. Available from: 10.1001/jamaoncol.2015.2510.

29. Morris E, Feig SA, Drexler M, Lehman C. Implications of Overdiagnosis: Impact on Screening Mammography Practices. Popul Health Manag. 2015;18(Suppl 1):3-11. Available from: 10.1089/pop.2015.29023.mor.

30. Spina S, Lombardi V, Terrasa S, Kopitowski K, Villalon G. Cuán precisos son los principales diarios de Argentina al informar sobre los métodos de prevención del cáncer de mama. Rev Argent Salud Pública. 2018;9(37):9-14. Available from: http://rasp.msal.gov.ar/rasp/articulos/volumen37/9-14. pdf.

31. Levinson W, Huynh T. Engaging physicians and patients in conversations about unnecessary tests and procedures: Choosing Wisely Canada. Canadian Medical Association Journal. 2014;186(5):325-326. Available from: 10.1503/cmaj.131674.

32. Allen JD, Bluethmann SM, Sheets M, Opdyke K, Gates-Ferris K, Hurlbert M, et al. Women's responses to changes in U.S. Preventive Task Force's mammography screening guidelines: results of focus groups with ethnically diverse women. BMC Public Health. 2013;13:1169-1169. Womens responses to changes in U.S. Preventive Task ForceHYPERLINK. Available from: 10.1186/1471-2458-13-1169.

33. Bouck Z, Pendrith C, Chen XK, Frood J, Reason B, Khan T, et al. Measuring the frequency and variation of unnecessary care across Canada. BMC Health Serv Res. 2019;19(1):446-446. Available from: 10.1186/s12913-019-4277-9.

34. Salgado MV, Kopitowski K, Barani M, Vietto V, Terrasa S. Sobreuso de mamografía para rastreo en un hospital académico de Buenos Aires. Rev Argent salud pública. 2016;7(27):7-11. Available from: http://rasp.msal.gov.ar/rasp/edicion-completa/RASP-XXVII.pdf.

35. Radhakrishnan A, Nowak SA, Parker AM, Visvanathan K, Pollack CE. Physician Breast Cancer Screening Recommendations Following Guideline Changes: Results of a National Survey. JAMA Intern Med. 2017:177(6):877-878. Available from: 10.1001/jamainternmed.2017.0453.

36. Riganti P, Discacciati V, Terrasa S, Kopitowski K. Factores motivacionales que influyen sobre las mujeres en la realizacin de mamografas de tamizaje de cncer de mama. Rev Argent Salud Pública. 2018;9(37):22-30.

37. Hersch J, Jansen J, Barratt A, Irwig L, Houssami N, Howard K, et al. Women's views on overdiagnosis in breast cancer screening: a qualitative study. BMJ. 2013;346:158-158. Available from: doi:10.1136/bmj.f158.

38. Mathioudakis AG, Salakari M, Pylkkanen L, Saz-Parkinson Z, Bramesfeld A, Deandrea S, et al. Systematic review on women's values and preferences concerning breast cancer screening and diagnostic services. Psychooncology. 2019;28(5):939-947. Available from: 10.1002/pon.5041. 
39. Barani M, Kopitowski K. Toma de decisiones compartidas: centrando los cuidados médicos realmente en nuestros pacientes. Rev Hosp Ital B Aires. 2013;33(2):60-64. Available from: https://www.hospitalitaliano.org.ar/multimedia/archivos/noticias_attachs/47/documentos/14745_60-64-HI33-2-2BARANI_A.pdf.

40. Stacey D, Légaré F, Lewis K, Barry MJ, Bennett CL, Eden KB, et al. Decision aids for people facing health treatment or screening decisions. Cochrane Database of Systematic Reviews. 2017;4:CD001431. Available from: 10.1002/14651858.CD001431.pub5.

41. Ivlev I, Hickman EN, Mcdonagh MS, Eden KB. Use of patient decision aids increased younger women's reluctance to begin screening mammography: a systematic review and meta-analysis. Journal of General Internal Medicine. 2017;32(7):803-812. Available from: 10.1007/s11606-017-4027-9.

42. Hersch J, McGeechan K, Barratt A, Jansen J, Irwig L, Jacklyn G, et al. How information about overdetection changes breast cancer screening decisions: a mediation analysis within a randomised controlled trial. BMJ open. 2017;7(10):e016246. Available from: 10.1136/bmjopen-2017-016246. 